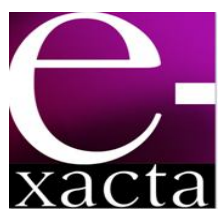

ISSN: 1984-3151

\title{
DETERMINAÇÃo dE ÉSTERES ETílICOS DE ÁCIDOS GRAXOS POR ESPECTROSCOPIA FT-NIR E CALIBRAÇÃO MULTIVARIADA EM REAÇÕES DE TRANSESTERIFICAÇÃO
}

\author{
Determination Of FAtTy ACIDS EthyL ESTERS by Ft-NiR \\ SPECTROSCOPY and Multivariate Calibration IN \\ TRANSESTERIFICATION REACTION
}

\author{
Djéssica Tatiane Raspe"; Vitor Augusto Garcia²; Camila da Silva ${ }^{3}$
}

1 Mestranda em Bioenergia, Universidade Estadual de Maringá. Umuarama, PR. Tecnóloga em Alimentos, Universidade Estadual de Maringá, PR. 2012.. djessicaraspe@hotmail.com.

2 Doutorando em Engenharia de Alimentos, Universidade de São Paulo. Pirassununga, SP. Mestre em Agronomia. Universidade Estadual de Maringá, 2011. garcia.vitoraugusto@gmail.com.

3 Doutora em Engenharia Química. Universidade Estadual de Maringá, 2009. Professora do Departamento de Tecnologia, Universidade Estadual de Maringá, Umuarama, PR. camiladasilva.eq@gmail.com.

Recebido em: 21/02/2013 - Aprovado em: 20/04/2013 - Disponibilizado em: 11/06/2013

RESUMO: O presente trabalho teve como objetivo estimar a concentração de ésteres etílicos de ácidos graxos (EEAG) em amostras da alcoólise de óleo de soja pela aplicação da espectroscopia FT-NIR associada à calibração multivariada. A amostra estudada foi obtida utilizando hidróxido de sódio como catalisador, em diferentes condições reacionais (temperatura, razão molar óleo, etanol, percentual de catalisador e tempo de reação), com o objetivo de obter amostras em diferentes concentrações de EEAG, a serem utilizadas nas etapas de calibração e validação da metodologia. Este trabalho utilizou a cromatografia gasosa com detector de ionização de chama (CG/DIC) como metodologia de referência e o método de mínimos quadrados parciais (PLS) no tratamento quimiométrico dos dados. Reporta uma alta correlação $(r>0,99)$ entre os dados obtidos pelo método FT-NIR/PLS e reportados pelo método CG/DIC. O modelo quimiométrico desenvolvido apresentou erro médio quadrático de 2,11 e 3,26 para as etapas de calibração e validação da metodologia. Além disso a capacidade de predição da metodologia FT-NIR/PLS foi testada com grupo independente de amostras, obtidas da alcoólise de óleo de soja. Os resultados obtidos demonstram que o método proposto apresenta-se como uma promissora técnica para avaliar o rendimento de reações de alcoólise de óleos vegetais, o que possibilitará o monitoramento de reações para produção de biodiesel.

PALAVRAS-CHAVE: Ésteres etílicos. Transesterificação. FT-NIR. Calibração multivariada.

ABSTRACT: This study aimed to estimate the concentration of fatty acid ethyl esters (FAEE) in samples from the alcoholysis of soybean oil using FT-NIR spectroscopy associated with multivariate calibration. The samples were obtained using sodium hydroxide as a catalyst at different reaction conditions (temperature, molar ratio oil:ethanol percentage of catalyst and reaction time), in order to obtain samples at different concentrations of FAEE, to be used in steps calibration and validation of the methodology. Gas chromatography with flame ionization detector (GC / FID) was used as the reference method and the method of partial least squares (PLS) used for treatment of chemometric data. It was reported a high correlation ( $r>0.99)$ between the data obtained by the method reported by FT-NIR/PLS and method GC / FID. The chemometric model developed showed mean square error of 2.11 and 3.26 for the steps of calibration and validation of the methodology. The predictive ability of the FT-NIR/PLS method was tested with independent group of samples obtained from the alcoholysis of soybean oil. The results show that the proposed method is presented as a promising technique for evaluating the performance of reactions of alcoholysis of vegetable oils, which will allow the monitoring of reactions for biodiesel production.

KEYWORDS: Ethyl esters. Transesterification. FT-NIR. Multivariate calibration. 


\section{INTRODUÇÃO}

O biodiesel pode ser definido como sendo um monoalquiléster de ácidos graxos derivado de fontes renováveis, como óleos vegetais e gorduras animais (CANAKCI; VAN GERPEN, 2001; MONYEM; VAN GERPEN, 2001; MONYEM; VAN GERPEN; CANAKC, 2001), obtido através de um processo de transesterificação, no qual ocorre a transformação de triglicerídeos em moléculas menores de ésteres de ácidos graxos (ENCINAR et al., 2002).

A reação de transesterificação ocorre em três etapas reacionais consecutivas e reversíveis, sendo obtidos diglicerídeos e monoglicerídeos como produtos intermediários dessa reação (MA; HANNA, 1999; FUKUDA; KONDO; NODA, 2001). A quantificação destes produtos, bem como dos triglicerídeos, glicerol e ésteres, é necessária buscando atender as especificações do biocombustível, bem como o monitoramento da reação, sendo que ela envolve análises laboratoriais sofisticadas. A Agência Nacional de Petróleo (ANP, 2013) especifica a determinação por técnicas cromatográficas que envolvem etapas complexas de preparo da amostra, como a derivatização e custos com padronização interna, além de não possibilitar a determinação de todos os compostos em apenas uma análise cromatográfica, devido a sinais sobrepostos. Outros métodos são aplicados envolvendo técnicas de cromatografia líquida de alta eficiência (KNOTHE; GERPEN; KRAHL, 2005), nas quais as etapas de derivatização são eliminadas. No entanto, observa-se o elevado consumo de solventes utilizados como fase móvel e longo tempo de verificação.

As análises quantitativas comumente utilizadas estão sendo substituídas por técnicas instrumentais, com o objetivo de aprimorar o controle de qualidade e o monitoramento de processos, como: ressonância magnética nuclear, espectroscopia no infravermelho, espectroscopia no visível/ultravioleta, etc. Tais técnicas aliam a velocidade de análise com uma boa qualidade de resultados (FERREIRA et al., 1999; GUARIEIRO et al., 2008; GUESTI, 2006; CORDEIRO, 2008). Entretanto, nessas técnicas instrumentais não é obtida uma informação direta do resultado, mas sim uma grande quantidade de sinais (curvas, picos) que podem ser tratados para uma possível quantificação das várias espécies presentes (FERREIRA et al., 1999). Nesse tratamento, a conversão da resposta instrumental no dado químico de interesse requer a utilização de técnicas de estatística multivariada, álgebra matricial e análise numérica. Essas técnicas se constituem no momento como a melhor alternativa para a interpretação de dados e para a aquisição do máximo de informação sobre o sistema em estudo (FERREIRA et al., 1999).

A calibração multivariada é a maneira mais efetiva para analisar diversos compostos simultaneamente. Sua habilidade de resolver misturas de diversos compostos com espectros sobrepostos tem sido amplamente utilizada. A principal vantagem se encontra na rápida determinação dos componentes na mistura, evitando separação prévia, o que geralmente é necessário no caso de sinais sobrepostos (FERREIRA et al., 1999; NUNES, 2008). Além disso, quando associados às técnicas espectroscópicas, são relativamente simples, permitem uma análise precisa, além da não destruição da amostra e não geração de resíduos nocivos ao meio ambiente (SARAIVA, 2007; NUNES, 2008; OLIVEIRA et al., 2004).

Dentro desse contexto, este trabalho teve por objetivo a aplicação da espectroscopia de infravermelho próximo com transformada de Fourier (FT-NIR) associada à calibração multivariada na quantificação de ésteres etílicos de ácidos graxos (EEAG) obtidos 
pela reação de transesterificação de óleo de soja em etanol.

\section{Metodologia}

Como matriz de calibração foram utilizadas amostras da reação de transesterificação de óleo de soja (Marca Soya) e etanol (Merck), usando hidróxido de sódio (Nuclear) como catalisador. Nas análises por cromatografia gasosa, utilizou-se heptano (F. Maia) como solvente e metil heptadecanoato (Sigma Adrich) como padrão interno.

As amostras do meio reacional de transesterificação (em base livre de etanol) foram obtidas conforme metodologia proposta por Faccio (2004). As reações foram conduzidas em diferentes condições reacionais, sendo obtidos modelos com percentuais de ésteres em intervalos de concentração de 20 a 97\%. Das 98 amostras estudadas, 66 foram utilizadas para calibração da metodologia e 32 para validação interna. Esta última foi testada com um grupo independente de 10 amostras.

A concentração de EEAG nas amostras foi determinada conforme a Normativa Europeia EN 14103 (2003). Para o preparo das amostras, foram utilizados os procedimentos descritos por Silva (2009).

As análises por cromatografia gasosa foram realizadas utilizando um cromatógrafo a gás Agilent GC 7890 A em triplicata equipado com uma coluna capilar RTX - Wax $(30 \mathrm{~m} \times 0,25 \mathrm{~mm} \times 0,25 \mu \mathrm{m})$. Perante as seguintes condições cromatográficas: temperatura inicial da coluna de $120^{\circ} \mathrm{C}$, permanecendo por 2 minutos nessa condição, aumentando-se a temperatura a uma taxa de $10^{\circ} \mathrm{C} /$ minuto até $180^{\circ} \mathrm{C}$, aumentando novamente a uma taxa de $5^{\circ} \mathrm{C} /$ minuto até $210^{\circ} \mathrm{C}$, permanecendo 1 minuto nesta condição e, finalmente, aumentando-se a uma taxa de $15^{\circ} \mathrm{C}$ /minuto até $230^{\circ} \mathrm{C}$, permanecendo assim por 2 minutos. Hélio foi utilizado como gás de arraste com a temperatura do injetor e detector em $250^{\circ} \mathrm{C}$ e a taxa de split de 1:50.

Determinado o percentual de EEAG nas amostras, realizou-se a sua medida em espectrofotômetro FTNIR N200 (Büchi) em triplicata. As medidas foram realizadas em temperatura de $20^{\circ} \mathrm{C} \pm 1{ }^{\circ} \mathrm{C}$, utilizando uma placa de petri de vidro (altura de $1,7 \mathrm{~cm} e$ diâmetro de $9 \mathrm{~cm}$ ), cobrindo uma região espectral de 1000 a $2500 \mathrm{~nm}$.

A análise quimiométrica dos dados obtidos foi realizada no Software NIR Cal 4.21, o qual avalia o melhor método quimiométrico para regressão dos dados, bem como o tratamento dos espectros de absorção (quando necessário). Os métodos propostos foram avaliados com base no erro médio quadrático (RMSE), determinado pela Eq. 1:

$$
\text { RMSE }=\sqrt{\sum_{i=1}^{n} \frac{\left(y_{p}-y_{\text {REF }}\right)^{2}}{N}}
$$

onde: " $\mathrm{y}_{\mathrm{p}}$ "e " $\mathrm{y}_{\mathrm{REF}}$ " denotam o valor previsto e o valor de referência, respectivamente. $O$ erro padrão relativo é designado como RMSEC (\%) para a calibração e RMSEP (\%) para a validação (predição).

\section{Resultados e Discussões}

Na Figura 1 é apresentado o espectro de absorção com pré-tratamento na região do Infravermelho Próximo para as amostras de calibração. Esta foi avaliada considerando a correlação entre o percentual de EEAG predito pelo FT-NIR e o percentual determinado pelo método cromatográfico de referência. 


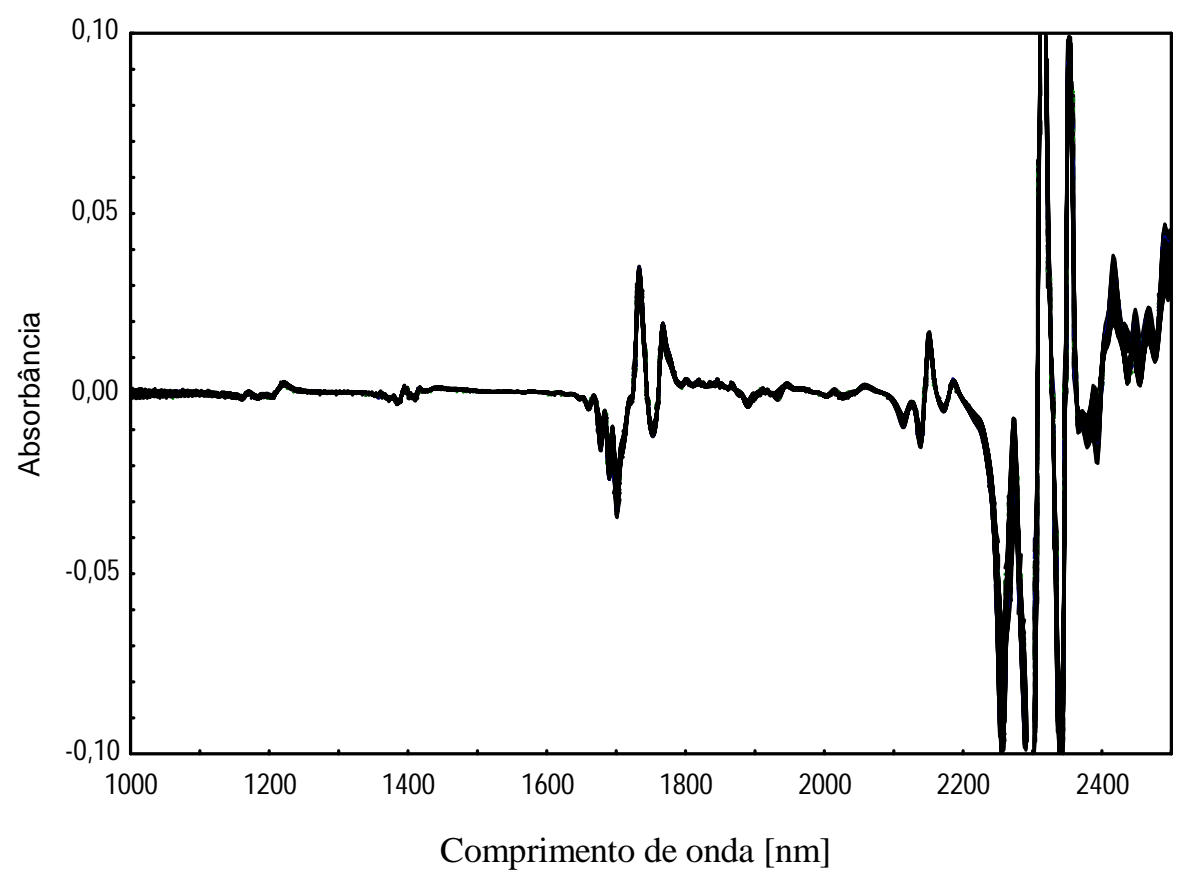

Figura 1 - Espectros das amostras obtidos por FT-NIR após tratamento.

No tratamento quimiométrico dos dados, avaliou-se o método dos mínimos quadrados parciais (PLS) para regressão dos dados para o espectro obtido na região de 1000 a $2500 \mathrm{~nm}$. O tratamento dos dados espectrais é necessário para evitar as flutuações de linha de base. Vários métodos foram testados sendo os seus resultados apresentados na TAB. 1.

Tabela 1

Resultados para validação de metodologia de predição do teor de EEAG (\%), utilizando diferentes métodos de pré-tratamento.

\begin{tabular}{cccc}
\hline Parâmetro & $\begin{array}{c}\text { Correção da Linha de Base } \\
\mathrm{a}\end{array}$ & $\begin{array}{c}\mathrm{a}^{+} \text {Suavização SG } \\
\text { b }\end{array}$ & $\begin{array}{c}\mathrm{b}+1^{\text {a }} \text { Derivada } \\
\mathrm{c}\end{array}$ \\
\hline Fatores primários & 8 & 8 & 8 \\
$\mathrm{R}^{2}$ calibração & 0,95 & 0,95 & 0,98 \\
$\mathrm{R}^{2}$ validação & 0,93 & 0,93 & 0,96 \\
RMSEC (\%) & 5,15 & 5,17 & 2,11 \\
RMSEP (\%) & 4,54 & 4,56 & 3,26 \\
Consistência & 113,53 & 113,53 & 86,03
\end{tabular}

a Obtida utilizando como referência o espectro sem tratamento.

b Suavização por Savitzky-Golay

c 1a derivada obtida pelo método de Savitzky-Golay

Os menores valores para o RMSEC e RMSEV foram obtidos utilizando 8 fatores primários para o pré- tratamento por $1^{\text {a }}$ derivada pelo método de SavitzkyGolay com correção da linha de base e suavização. A 
correlação linear feita entre as metodologias apresentou valores de 0,98 e 0,96 para calibração e validação. A consistência é definida como a percentagem de erro padrão da estimativa (SEE), dividido pelo erro padrão de previsão (SEP) e deve ser o mais próximo possível de 100, com valores aceitáveis entre 80 e 110. O valor de consistência de 86,03 encontrada neste trabalho suporta a qualidade do método desenvolvido.

A Figura 2 apresenta a correlação entre o método FTNIR e a metodologia analítica de referência. Baptista et al. (2008) reportam a eficácia da aplicação de calibração multivariada acoplada à espectroscopia NIR para a quantificação de ésteres metílicos obtidos da transesterificação de óleo de palma, soja e canola. O método PLS é aplicado para o espectro com prétratamento (1. a derivada pelo método de SavitzkyGolay), obtendo erro padrão relativo de 1,0 e 1,1 para as etapas de calibração e validação. A aplicação do método PLS e redes neurais artificiais na quantificação de ésteres etílicos do óleo de soja são reportadas por Silva et al. (2007). Os autores utilizam como técnica analítica a espectrofotometria de absorção molecular e obtêm RMSEP de 4,09 e 2,45 para o método PLS e para o método utilizando redes neurais respectivamente para a etapa de predição.

Killner et al. (2011), visando elaborar um modelo de regressão PLS usando espectroscopia NIR para monitoramento on-line de ésteres metílicos, obtidos através da reação de transesterificação, obtiveram um erro médio quadrático na etapa de calibração (RMSEC) e validação (RMSEV) de 1,12 e 1,56 respectivamente. Richard et al. (2011), estudando o monitoramento on-line da reação de transesterificação entre triglicerídeos e etanol por espectroscopia no infravermelho próximo (NIR) -, utilizando como metodologia de referência a cromatografia gasosa (CG), constataram em seu trabalho que o melhor modelo de calibração foi o que apresentou um RMSEC médio de 1,09 e RMSEV de 1,32 (\% de conversão).

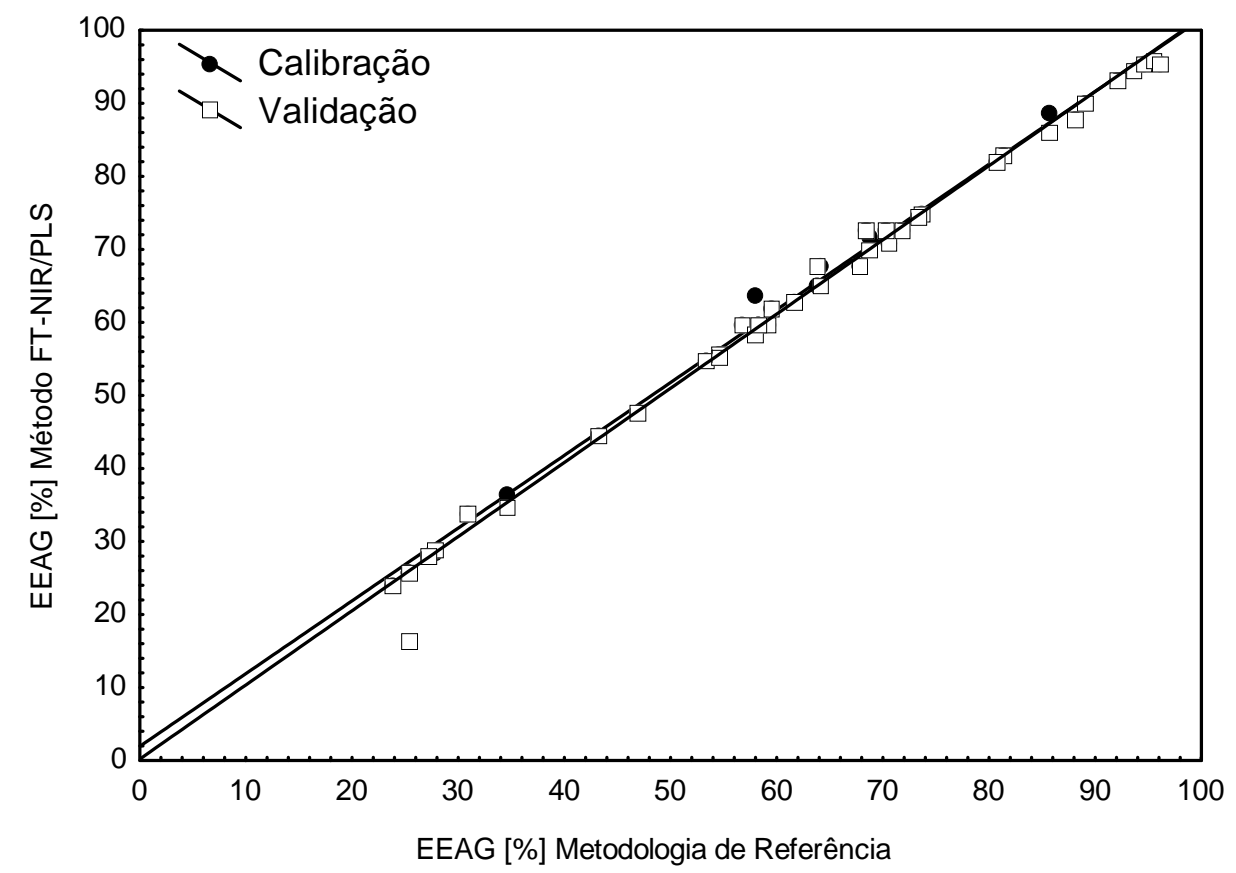

Figura 2 Correlação entre o percentual de EEAG predito pelo método FT-NIR/PLS e observada pelo método de referência. 
Na Figura 3 são apresentados os resultados para aplicação da espectroscopia FT-NIR na avaliação do rendimento da transesterificação de óleo de soja em etanol, a qual relaciona o percentual de EEAG obtido pela metodologia de referência e 0 obtido pelo FT-NIR associado à calibração multivariada Verifica-se que a metodologia proposta é eficaz a fim de predizer o percentual de ésteres etílicos, devido aos baixos desvios.

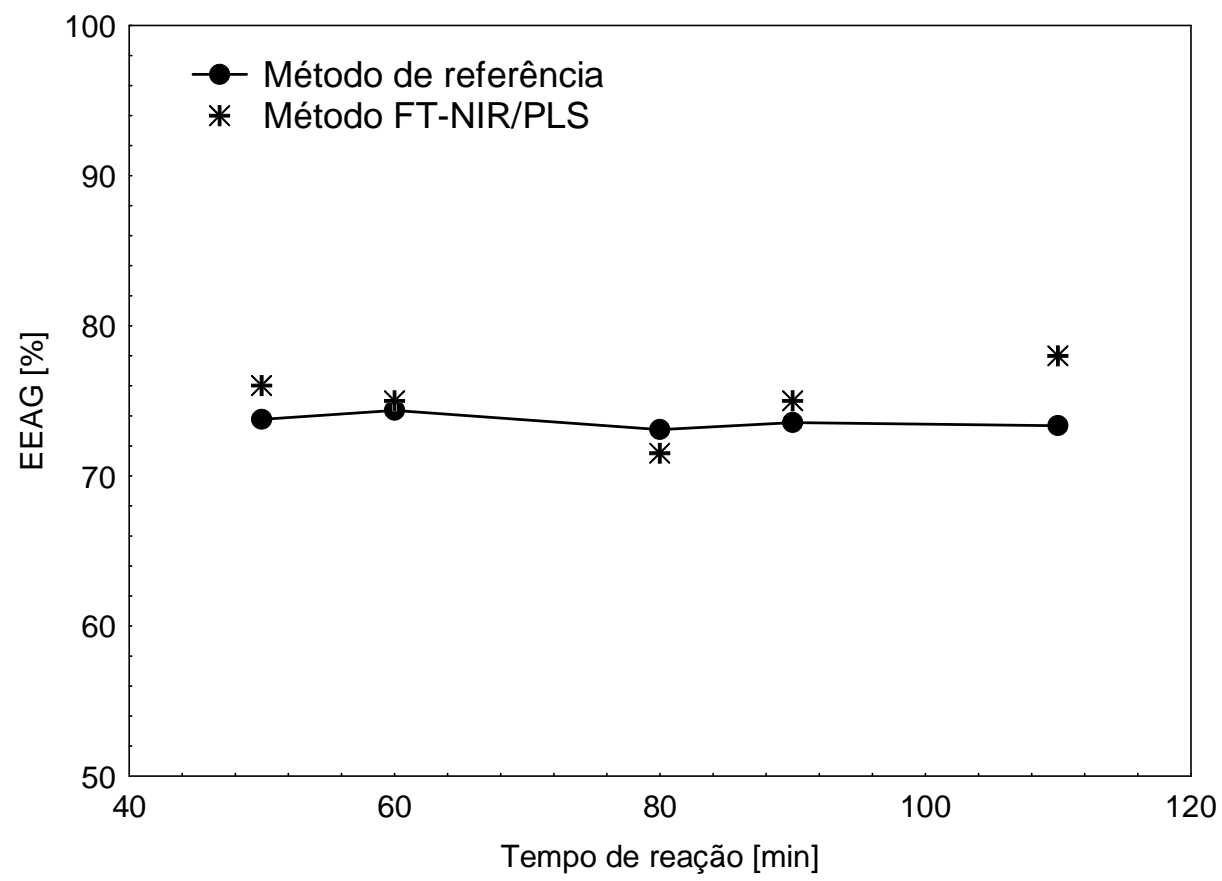

Figura 3 - Correlação entre a conversão da reação predita pelo método FT-NIR/PLS e observada pelo método de referência.

\section{CONCLUSÕES}

Um método simples e rápido para determinação do percentual de ésteres etílicos de ácidos graxos, a partir da esterificação e transesterificação de óleo de soja em etanol, foi proposto, usando espectroscopia FT-NIR e calibração multivariada.

As etapas de calibração e validação da metodologia apresentaram valores de RMSEC e RMSEV de 2,11 e 3,26 , respectivamente. Os resultados demonstram que a espectroscopia FT-NIR é uma ferramenta valiosa para predição do percentual de ésteres etílicos a partir da transesterificação de óleo de soja em etanol com baixos desvios em relação à metodologia de referência. Isso demonstra a possibilidade de utilização de tal metodologia para o monitoramento de reações de transesterificação.

\section{AgRADECIMENTOS}

Ao CNPq, pelo suporte financeiro, e à Universidade Estadual de Maringá, Campus Umuarama, pela infraestrutura. 


\section{REFERÊNCIAS}

ANP. Agência Nacional de Petróleo. <www.anp.gov.br>. Acesso em: jan. 2013.

BAPTISTA, P. et al. Multivariate Near Infrared Esters Content in Biodiesel. Analytica Chimica Acta, Amsterdam, 607(2), 153-159, 2008.

CANAKCI, M.; VAN GERPEN, J. Biodiesel production from oils and fats with high free fatty acids. Transactions of the ASAE, St. Joseph, 44(6), 14291436, 2001.

CORDEIRO, C. S. Compostos lamelares como catalisadores heterogêneos em reações de Transesterificação Metílica. 2008. 147 f. Tese (Doutorado em Química) - Universidade Federal do Paraná, Curitiba, 2008.

ENCINAR, J. M.et al. Biodiesel fuels from vegetable oils: transesterification of Cynara cardunculus L. oils with ethanol. Energy \& Fuel, London, 16(2), 443-450, 2002.

EN14103: Fatty acid methyl esters (FAME) Determination of ester and linolenic acid methyl esters contents. Asociación Española de Normalización y Certificación. Madrid, 2003.

FACCIO, C. Estudo da produção de ésteres etílicos a partir da alcoólise de óleos vegetais. 2004. 70 f. Dissertação (Mestrado em Engenharia de Alimentos) Universidade Regional Integrada do Alto Uruguai e das Missões, Erechim, 2004.

FERREIRA, M. M. C. et al. Quimiometria l: calibração multivariada, um tutorial. Química Nova, São Paulo, 22(5), 724-731, 1999.

FUKUDA, H.; KONDO, A.; NODA, H. Biodiesel Fuel Production by Transesterification of Oils. Journal of Bioscience and Bioengineering, Osaka, 92(5), 405416, 2001.

GUARIEIRO, L. L. N. et al. Metodologia analítica para quantificar 0 teor de Biodiesel na mistura Biodiesel:Diesel utilizando espectroscopia na região do Infravermelho. Química Nova, São Paulo, 31(2), 421-426, 2008.

GUESTI, G. F. Estudo de Catalisadores para a obtenção de Biodiesel por Transesterificação e Determinação do Rendimento por Espectroscopia Raman. 2006. 55 f. Dissertação (Mestrado em Química) - Universidade de Brasília, Brasília, 2006.

KILLLER, M.H.M.; ROHWEDDER, J.J.R; PASQUINI, C. A PLS regresion model using NIR spectroscopy for on-line monitoring of the biodiesel production reaction. Fuel, 90(1), 3268-3273, 2011.

KNOTHE, G.; GERPEN, J. V.; KRAHL, J. The Biodiesel Handbook. AOCS Press: Champaign, IL, USA, 2005.

MA, F.; HANNA, M. Biodiesel production: a review. Bioresource Technology, Essex, 70(1), 1-15, 1999.

MONYEM, A.; VAN GERPEN, J. H. The effect of biodiesel oxidation on engine performance and emissions. Biomass and Bioenergy, Oxford, 20(4), 317-325, 2001.

MONYEM, A.; VAN GERPEN, J. H.; CANAKCI, M. The effect of timing and oxidation emissions from biodiesel-fueled engines. Transactions of the ASAE, St. Joseph, 44(1), 35-42, 2001.

NUNES, P. G. A. Uma nova técnica para seleção de variáveis em calibração multivariada aplicada às espectroscopias UV-VIS e NIR. 2008. 106 f. Tese (Doutorado em Química) - Universidade Federal da Paraíba, João Pessoa, 2008.

OLIVEIRA, F. C. C. et al. A escolha da faixa espectral no uso combinado de métodos espectroscópicos e quimiométricos. Química Nova, São Paulo, 27(2), 218225, 2004.

RICHARD, R. et al. On-line Monitoring of the Transesterification Reaction between Triglycerides and Ethanol Using Near Infrared Spectroscopy Combined with Gas Chromatography. Bioresource Technology, 102(12), 6702-6709, 2011.

SARAIVA, J. O. Avaliação da qualidade de biodiesel por espectroscopia FTIR e FTNIR associadas à quimiometria. 2007. 175 f. Dissertação (Mestrado em Química) - Universidade de Brasília - UnB, 2007.

SILVA, C. Produção Contínua de Biodiesel por transesterificação não catalítica de óleo de soja. 2009. 180 f. Tese (Doutorado em Engenharia Química) Universidade Estadual de Maringá, 2009.

SILVA, C.; et al. Aplicação de Ferramentas Quimiométricas na Reação de Transesterificação de óleo de soja. In: XX Congresso Latino Americano de Óleos e Gorduras, Anais... Florianópolis, Santa Catarina, 2007. 